\title{
Intervención multidisciplinaria del trabajador social en el área gerontológica
}

\author{
Multidisciplinary intervention of the social worker in the gerontological area
}

Cristhian Michael Bailón Anchundia

Universidad Técnica de Manabí - Ecuador

Portoviejo, Ecuador

cbailon9120@utm.edu.ec

Ligia Estela Loor Lino

Universidad Técnica de Manabí - Ecuador

Portoviejo, Ecuador

ligia.loor@utm.edu.ec

\section{RESUMEN}

La vulneración de las personas adultas mayores requiere atención especializada por organismos que aporten en su calidad de vida, como respuesta a la problemática que puede complicarse en los ámbitos sociales. El objetivo de esta investigación consistió en identificar la intervención multidisciplinaria del trabajo social en el área gerontológica en el centro Gerontológico Guillermina Loor de Moreno de la ciudad de Portoviejo, Ecuador. El estudio fue de tipo descriptivo, con un enfoque cualitativo, como fuente de recopilación de información se utilizó la técnica de la entrevista y como instrumento la guía de entrevista que se aplicó a la trabajadora social del centro gerontológico. Los resultados demuestran que la participación de la trabajadora social con el equipo multidisciplinario en el proceso de valoración de la persona adulta mayor permite realizar acciones de organización, programación y evaluación de las actividades del centro por lo que se implementan actividades complementarias como realizar la identificación, mapeo y coordinación de redes con otras instituciones, que de manera eficaz permiten a las personas adultas mayores en situación de calle incorporase al centro gerontológico. La gestión interinstitucional que realiza el trabajo social en el centro gerontológico le permite integrar estrategias de prevención y promoción de la salud buscando acciones integrales de manera paliativa, la cual sigue procesos sistemáticos de evaluación.

Palabras claves: Intervención Multidisciplinaria; Trabajo social; Adulto mayor; Centro Gerontológico.

\section{ABSTRACT}

The vulnerability of older adults requires specialized attention by agencies that contribute to their quality of life, as a response to the problem that can be complicated in social settings. The objective of this research was to identify the multidisciplinary intervention of social work in the gerontological area at the Guillermina Loor de Moreno Gerontological Center in the city of Portoviejo, Ecuador. The study was descriptive, with a qualitative approach, the interview technique was used as a source of information collection and the interview guide was applied to the social worker of the gerontological center as an instrument. The results show that the participation of the social worker with the multidisciplinary team in the assessment process of the elderly person allows the organization, programming and evaluation of the center's activities, which is why complementary activities are implemented, such as the identification, mapping and coordination of networks with other institutions, which effectively allow the elderly people in street situations to join the gerontological center. The interinstitutional management carried out by social work in the gerontological center allows it to integrate prevention and health promotion strategies, seeking comprehensive palliative actions, which follow systematic evaluation processes.

Keywords: Multidisciplinary Intervention; Social Work; Elderly; Gerontological Center. 


\section{Introducción}

El envejecimiento es un proceso biológico normal que va acompañado de ciertos cambios estructurales y también funcionales los cuales aparecen en el transcurso del tiempo, es decir, que no tiene que ver precisamente con la afección o condición de enfermedad, por lo que es necesario entender que envejecer es la creciente incapacidad del cuerpo de una persona de mantenerse por sí solo y realizar las cosas que hacía antes. Para Uribe, envejecer es una circunstancia natural de la vida en la cual "este proceso inicia a los 60 años y termina en el momento en el que la persona fallece” (2010, p. 247).

Se debe precisar que cada individuo lleva un proceso diferente de otro, si bien se debe alcanzar determinada edad para considerarse envejecimiento natural, existen factores que pueden acelerar esta situación, por ello es "necesario entender que esta etapa incide en la persona, la familia y la comunidad, y esto conlleva a desarrollar planes de acciones integrales y solidarias, que contribuyan a valorizar el rol de las personas mayores en la sociedad" (Paredes y Monteiro, 2019, p. 90)

Frente a esta situación surge la necesidad de definir la gerontología como ciencia que se dedica al estudio diferentes aspectos del envejecimiento en los seres humanos de edad avanzada, que establece pautas importantes para que los profesionales puedan implementar estrategias con enfoques pragmáticos basados en resultados que puedan ser evaluados, tal como lo expresan Martínez et al., quienes establecen que "es la ciencia destinada al estudio del envejecimiento de los seres vivos y de las personas en todos sus aspectos: biológico, psicológico, socio - económico y cultural" (2013, p. 127). Por esta razón aprender a envejecer representa captar rápidamente lo que puede brindar la vida en la vejez, aceptando las limitaciones normales para la edad, en este sentido, Cárdenas y Cedeño, definen "un lugar para tratar a las personas de la tercera edad, en donde además de controlar su salud se pueda estimular y fomentar la inclusión social de adultos mayores" (2018, p. 4).

Un adulto mayor por su condición de edad, puede significar para muchos como aquel individuo que luego de una etapa biológica, evoluciona de manera natural alcanzando un determinado nivel de conocimientos, procesos llamados experiencias, en la cual se distingue sabidurías, no obstante, para Donizete y Dutra, (2006) la prevención geriátrica debe iniciar desde los 60 años, considerada una edad adulta, pero también en la adolescencia e incluso durante el embarazo.

Por cuanto, una persona a esa edad es importante, sobre todo en el ámbito familiar y social, debido a que las personas mayores garantizan la transmisión del saber a través de todas las experiencias de la vida que componen una fuente de conocimiento que las futuras generaciones deben aprovechar. Cada adulto mayor representa una pieza primordial para la sociedad, han estado presentes en distintos momentos cruciales lo que permitió que tuvieran un alto conocimiento y experiencias en diversas áreas que sirvió como base sólida para construir en la historia y desarrollo del mundo en que vivimos.

Para Sepúlveda, (2012) la atención del adulto mayor puede ser una herramienta útil la gerontología misma, la cual manifiesta que "Es una disciplina que se concibe como una nueva dimensión derivada de la psicología, gerontología y la sociología que estudia los problemas políticos, económicos, sociales y sanitarios del entorno relativo al adulto mayor" (p. 3). Además, es multidisciplinaria al 
incluir varias ciencias y profesiones dirigidas al estudio de la vejez, de este modo, la gerontología social estudia los aspectos biológicos y psicológicos de las personas mayores, porque, para los profesionales que inician una carrera laboral relacionada con el bienestar del colectivo se constituye como la intención de identificar los problemas y desafíos que enfrentan el adulto mayor en su entorno social con el objetivo de proporcionar acciones influyan en la mejora de la calidad de vida de las personas mayores.

Por otra parte, Díaz afirma que "la principal generación de teorías basadas en gerontología social inicio con la interacción de la gerontología y la psicología social” (2011, p. 52). Por esta razón, tiene un amplio carácter individualista referente a la psicología social sobre la adaptación o inadaptación del adulto mayor en el declive de sus actividades funcionales que es esencialmente la capacidad de interacción del individuo en su entorno social, es por ello, que su estudio se centra en acudir a elementos de nivel micro como normas, principios y grupos de referencia para incorporar medidas de gestión en función a la atención que requieren las personas vulnerables como lo son los adultos mayores.

No obstante, para Varela cada uno de los elementos epistemológicos, teóricos y metodológicos que integran la gerontología social, están asociados al análisis y comprensión respecto al envejecimiento, por tanto, se fundamentan en cuatro enfoques teóricos principales que están sujetos a la permanencia de la construcción social del adulto mayor como es: "Funcionalismo Estructural, Interaccionismo Simbólico, Intercambio en la Vejez y Economía Política que dan sentido a la ejecución de acciones orientado al fortalecimiento del vínculo social y valor el ejercicio de la ciudadanía de las personas mayores" (2000, p. 13).

Por esta razón, los profesionales implicados en el estudio de la gerontología social como los adultos mayores tienen como propósito incluir por medio de la aplicación y creación de directrices la coordinación, organización y programación de actividades que contribuyan a que los adultos mayores tengan acceso a una atención de salud como participación social adecuada. Esto abordado con diferentes disciplinas que se organizan en un objetivo en común.

Para Franco y Cárdenas (2021) generalmente la intervención del trabajo social en diferentes áreas es planificar, programas, y diseñar objetivos y metas destinados a que los adultos mayores tengan una vida digna conforme los principios y valores reflejados en la sociedad, puesto que, su actuar busca mejorar la calidad de vida de este grupo de edad avanzada teniendo como objetivo lograr un bienestar colectivo con base a la respuesta y las demandas que presentan este grupo de personas.

Desde esta perspectiva, las estrategias de intervención multidisciplinarias es uno de los pilares fundamentales, ya que se refiere a intentar reducir tensiones existentes en el seno de la familia del adulto mayor, porque una de sus mayores necesidades es ser escuchados y lamentablemente ese requerimiento es levemente atendido, en este sentido, “la evaluación repetida de la fragilidad y la trayectoria de la fragilidad del individuo podría proporcionar un medio para anticipar un mayor deterioro de la salud y la mortalidad y, por lo tanto, podría respaldar la toma de decisiones clínicas" (Stolz et al., 2021, p. 1625).

Es necesario resaltar que la intervención multidisciplinaria que realice el Trabajador social debe ser siempre orientada al bienestar de los grupos prioritarios, velando por el cumplimiento de sus 
derechos y por la corresponsabilidad de la familia, estado y sociedad, y de eso se desprende que el trabajador social brinde propuestas a las personas adultas que propicie el fortalecimiento de los lazos familiares, mejorando las condiciones de vida de cada adulto mayor en el cumplimiento de las necesidades dando solución de los problemas sociales, identificando la realidad tal como es percibida sin ningún tipo de manipulación, acciones que son frecuentes en la actualidad, tal como señala Llarena \& Hine "manipular la tasa de envejecimiento y retrasar la aparición de enfermedades relacionadas con el envejecimiento han sido la composición de las actividades médicas, científicas y pseudocientíficas a lo largo de la historia" (2021, p. 1554). Es decir, que intervenir en el área gerontológica requiere procesos que busquen un propósito de mejoras en diferentes aspectos en el adulto mayor, y para ello el profesional debe estar en empoderamiento sistémico de la realidad en la cual interviene.

De acuerdo a lo que antecede en esta investigación, y por la relevancia que implica para la sociedad, se plantea la siguiente interrogante: ¿Cómo se realiza la Intervención social por parte de los trabajadores sociales en el área gerontológica? Para efecto la función que el profesional desarrolla en su actividad laboral, se considera el siguiente objetivo: Determinar la intervención multidisciplinaria del trabajo social en el Gerontológico Guillermina Loor de Moreno ubicado en la ciudad de Portoviejo, Ecuador.

\section{Metodología}

La ejecución de esta investigación fue de tipo descriptiva, se fundamentó con el enfoque cualitativo, que según (Sánchez, 2019, p. 105) “se sustenta en evidencias que se orientan más hacia la descripción profunda del fenómeno con la finalidad de comprenderlo y explicarlo a través de la aplicación de métodos y técnicas derivadas de sus concepciones y fundamentos epistémicos”. También se apoyó en fuentes fidedignas de información, utilizando el método lógico científico, bibliográfico, inductivo deductivo, lo que permitió abordar la problemática desde lo generar a lo particular, acompañado de análisis síntesis en el cual se obtuvo información en revistas de alto impacto, lo que fue relevante sobre la problemática investigada, también se utilizó la técnica de la entrevista y como instrumento la guía de entrevista que se aplicó a la trabajadora social, y al equipo interdisciplinar del centro gerontológico Guillermina Loor de Moreno del cantón Portoviejo.

\section{Resultados}

Luego de aplicar la técnica de la entrevista a la trabajadora social del centro gerontológico, Guillermina Loor de Moreno (CGGLM), del cual su objetivo es Identificar la Intervención multidisciplinaria del Trabajo social en el área Gerontológica, se obtuvieron los siguientes resultados.

Para el abordaje en la recopilación de datos, se preguntó a la trabajadora social sobre los diferentes profesionales que intervienen al adulto mayor desde la incorporación al centro gerontológico, así como en las acciones ambulatorias que se realizan con el fin de llegar con atención a quienes por distintos motivos no están de manera permanente.

(...) El centro gerontológico al ser una institución que busca el bienestar del adulto mayor, sigue una serie de procedimientos en ese fin, en la cual el equipo multidisciplinario la integran profesionales en diferentes áreas con énfasis en aspectos de; salud, estado 
funcional y mental, también nutricional, social, económico, entre otros; por lo que es importante y necesario disponer de un equipo que aporte al mejoramiento y calidad de vida del adulto mayor desde cada área mencionada (Trabajadora social).

Identificando que dentro del CGGLM, su área es lo social, ¿qué acciones realiza usted dentro de sus competencias para el mejoramiento de la calidad de vida del adulto mayor en el centro gerontológico?

(...) Podría resumir que dentro de todas las acciones que como Trabajadora social realizo, está la de gestión, seguimiento, coordinación y otras que permiten dentro de mis funciones buscar diferentes medios alternos para intervenir en cualquier situación que se presente, no obstante, todas se sustentan en la de promoción, de la cual se busca que el envejecimiento activo y saludable sea una de las tareas significativas para la comprensión de la sociedad por cuanto permite involucrar a la comunidad en general como una base de responsabilidad en el cuidado del adulto mayor. Sin embargo, la coordinación interinstitucional permite precisamente poner en el propósito inicial de que por medio de diferentes profesionales se logren los objetivos en el adulto mayor (Trabajadora social).

Dentro de esta investigación se hace necesario preguntar, de qué manera involucra a la sociedad en el proceso de intervención en el adulto mayor,

(...) El CGGLM, implementa los espacios formativos, que consisten mediante la aplicación de talleres, charlas, así como foros, entre otros; que involucran directamente a las personas adultas mayores, con la comunidad y el equipo técnico, todo eso mediante una coordinación por parte de Trabajo social, en los que el propósito es la incorporación de contenidos para la atención integral de las personas adultas mayores, puesto que. la aportación proactiva de la familia, la asistencia en programas sociales, culturales, son identificados como formas y mecanismos que involucran la corresponsabilidad familiar y comunitaria (Trabajadora social).

Según el plan de intervención individual del adulto mayor, dentro de su rol profesional, ¿En qué aporta su accionar como Trabajadora social?

(...) Por medio de la participación con el equipo multidisciplinario en el proceso de evaluación de la persona adulta mayor, puesto que mi rol me permite realizar acciones de organización, programación y evaluación de las actividades del centro por lo que se implementan actividades complementarias como realizar la identificación, mapeo y coordinación de redes con otras instituciones, que de manera eficaz permitan a las personas adultas mayores en situación de calle incorporase al centro gerontológico (Trabajadora social).

Considerando que en dentro del centro gerontológico su accionar es implementar mejoras en la población vulnerable, ¿Cuál es su gestión con el equipo interdisciplinar del centro?

(...) Como trabajadora social unos de los procedimientos que realizo es la coordinación con otros profesionales en función de atención al adulto mayor, y cuando se realiza la elaboración de la ficha social precisamente se debe cooperar con otros compañeros para tener información precisa, de igual manera participo en la planificación general misma que se organiza con psicólogos que intervienen en el adulto, en resumen, puedo indicarle que 
con el equipo de profesionales existe organización de actividades, buena comunicación, adecuada interacción, decisiones y concesos con el fin de brindar al adulto mayor toda la atención pertinente según si situación (Trabajadora social).

Cuando un adulto mayor, presenta inconvenientes en el estado de salud, usted como profesional en trabajo social, ¿Qué acciones realiza para intervenir en el proceso de recuperación o prevención en esta situación?

(...) Dentro del proceso integral que el CGGLM realiza durante el ingreso de un adulto mayor, se encuentran los resultados de la valoración gerontológica la cual establece la condición especifica del adulto, las acciones que se deben seguir y los mecanismos directos de actuación, hacia la persona adulta mayor, lo que se le informa a su familia en caso de tenerla, todo este proceso se realiza mediante un informe semestral, con la finalidad de establecer diferentes estrategia de trabajo, sin embargo cuando se presentan inconvenientes de salud, se hace la gestión con el MSP quienes realizan la valoración y emiten recomendaciones que se siguen de manera específica. No obstante, los procesos de prevención que se realizan son el control constante del estado de salud, mediante la programación de jornadas en el centro de salud más cercano, o por pedidos expreso de la dirección sobre visitas medidas programadas (Trabajadora social).

La siguiente entrevista se aplica a la coordinadora del Centro Gerontológico con el fin de incluir aspectos relevantes para el desarrollo de la investigación, acción que permitirá cumplir con el objetivo:

¿Desde su criterio personal, como considera intervención de la trabajadora social cuando trabajada con usted?

Considero que la colega tiene excelentes criterios de intervención, y cuando lo hacemos en conjunto ella articular acciones con las familias y comunidad, lo cual resalto como positivo para este centro (Coordinadora del Centro Gerontológico).

¿Qué criterios considera usted relevantes de la trabajadora social que identifiquen su accionar en del centro gerontológico?

Considero entre tantas cosas que resalto de ella es la organización, el compromiso, responsabilidad, empatía todo esto se evidencia en el desempeño de las actividades que realiza, permitiendo lograr resultados propuestos (Coordinadora del Centro Gerontológico).

Continuando con la aplicación de la técnica en la investigación, se plantearon las siguientes interrogantes al psicólogo clínico para identificar desde su perspectiva las acciones que desarrolla la trabajadora social.

¿De qué manera trabaja con la Trabajadora social de la institución?

Cuando realizo la valoración individual del adulto mayor, coordino con la Trabajadora social los siguientes procesos a realizar como talleres de terapias, monitoreo de estado de las personas adultas, en donde es muy importante la gestión que la colega realiza porque ella organiza los días adecuados según la situación del adulto o los programas que se tiene previstos en la institución (Psicólogo clínico). 
En el proceso de intervención grupal que realizan en los adultos mayores ¿De qué manera aporta la Trabajadora social en la ejecución del proceso?

En el diseño y aplicación de las estrategias que se desarrollan, ella aporta con criterios técnicos para la interacción y valoración, permitiendo el logro de los objetivos planteados (Psicólogo clínico).

Finalmente se consideró oportuno entrevistar al Terapista Ocupacional, quien desempeña acciones en los adultos mayores, y por tener incidencia en el bienestar, se le plantearon las interrogantes:

¿De qué manera la trabajadora social participa en la ejecución de sus actividades profesionales?

Cuando voy a aplicar el proceso de evaluación de las capacidades y seguimiento del desempeño de las personas adultas mayores que ingresan al servicio gerontológico quien me colabora con información, del adulto mayor es la colega, pues considero que ella identifica cada situación del adulto mayor y lo pone de manifiesto para considerar cada situación individual por lo que trabajar de manera coordinada con ella es una buena acción (Terapista Ocupacional).

¿Durante la intervención que usted realiza en los adultos mayores, la trabajadora social participa de sus acciones?

No, cada profesional realiza su intervención, según la situación y naturaleza a intervenir, no obstante, si existe coordinación y gestión de parte de la colega, pues antes de realizar una actividad, reviso el informe que emite, para conocer si existe alguna situación importante a considerar. Debo resaltar que ella es muy atenta al momento de solicitarle su apoyo (Terapista Ocupacional).

\section{Discusión}

Abordar la problemática relacionada con la edad del ser humano puede convertirse en propósitos justo desde una perspectiva social, pues si bien, la naturaleza humana y su complejo sistema biológico nos direcciona a llegar en esta etapa con el transcurso de los años, no obstante, la implicación social es protagonista de establecer una diferencia entre una situación positiva o distinta Lo que permite una inserción de profesionales en Trabajo social en ese ámbito, quien debe intervenir para lograr el bienestar de las personas adultas desde ese criterio Nascimento et al., (2020) señalan que:

...cuidar a una persona mayor es una actividad difícil y requiere casi siempre dedicación total. El gran problema es que suele deberse a factores como: baja educación, estrés por sobrecarga física y emocional, falta de experiencia, edad avanzada o incluso falta de empatía; Se vuelve difícil proporcionar un apoyo adecuado (p. 110).

Lo que implica responsabilidades en el proceso, por medio de participación con un equipo interdisciplinario que aborde acciones en direccional objetivo común, el bienestar integral de los adultos mayores, considerando todo lo que pueda incidir en transcurso de la acción, de manera directa cuando se realiza valoraciones específicas de cada profesión basados en el cuidado que estos proporcionan al adulto mayor, así lo señalan (Ramos y García) “los cuidados de larga 
duración en la actualidad se confrontan con la necesidad de garantizar para las personas una calidad de vida que responda a sus deseos, es decir, a su participación en la definición y desarrollo de este proceso" (2021, p. 49).

En este sentido, todos los derechos establecidos por el Estado ecuatoriano tienen como objetivo eliminar los estereotipos de las personas en relación con su edad, etnia, género y origen, debido a que, la aplicación de cada norma tiene como finalidad que las personas más vulnerables tengan acceso a mantener una vida digna, justa, segura y con atención prioritaria conforme lo soliciten por parte de las organizaciones e instituciones del país.

Mientras que Sanhueza et al., (2005) menciona que los adultos mayores representan pilares en que se fundamenta la sociedad, debido a que, en todas las etapas de su vida se han adaptado a diversos cambios políticos, económicos, sociales entre otros, que les permitió incorporar conocimientos que fueron concedidos de forma popular a sus descendientes que ha logrado influir en la forma en la que vive con el anhelo de que nuestra sociedad tenga un mejor presente y un esperado mejor futuro. Por esta razón, es necesario que se sustente su bienestar y protección en relación con los cambios naturales que enfrentan las personas mayores lo cual radica en la disminución de sus actividades funcionales por medio de la atención integral por parte de los organismos públicos y privados para que le proporcione el acceso a una mejor calidad de vida conforme a la gestión de los procesos de actividad fisiológica, psicológico, mental y espiritual.

Es importante destacar que como sociedad debemos atender a los cambios que una persona atraviesa, es decir de joven a adulto mayor, por lo que se considera indispensable establecer acciones que busquen paliar esos efectos en las personas adultas mayores, con disciplinas que se dediquen a comprender el proceso y las alternativas que se deben ejecutar durante este.

Es importante señalar que cada profesional gestiona su procedimiento según las competencias asumidas, sin embargo, esta debe responder a los objetivos comunes, que permitan viabilizar cambios positivos en los adultos que se intervienen, según Pérez y Moya durante este proceso "se registran los recursos y posibilidades de la persona, se valora la necesidad de servicios y finalmente, se elabora un plan de cuidados progresivos, continuados y coordinados dirigido a satisfacer las necesidades del paciente y sus cuidadores" (2019, párr. 2). Mismos que deben responder a la situación identificada, las acciones desarrolladas y la coordinación planteada por el profesional a cargo de la situación planteada.

Responder a la situación que vive el adulto mayor sin una protección adecuada es solo la iniciativa básica que como sociedad debe enfatizarse, con el adecuado uso de estrategias y cuidados de quien tenga la responsabilidad de asumir esa función de cuidar al adulto mayor, comprometido con el fin propuesto, con el adecuado uso y disposición de recursos, "aunque resulta difícil lograr, la meta final es que el cuidador de adultos mayores fuera o dentro del asilo, tenga las suficientes herramientas físicas y emocionales para que se conjugue la salud integral de ellos con la prevención y tratamiento" (Hernández, 2006, p. 43).

Si bien el cuidador resulta importante para el cuidado del adulto dentro de los centros gerontológicos, los profesionales que realizan intervenciones de manera sistemática son relevantes al momento que un adulto ingresa, no obstante, el Trabajador social según su rol permite realizar diferentes procedimientos, entre ellos la "evaluación preliminar de las personas 
adultas mayores, que solicitan el ingreso al centro residencial a través de la aplicación de fichas sociales" (Ministerio de Inclusión Económica y Social, 2018, p. 25). En este sentido, las acciones que realiza el profesional le permiten gestionar, programar evaluar, dar seguimientos para trabajar con diferentes profesionales en busca del propósito en común de atender e intervenir en el adulto mayor.

Desde este orden de ideas, “los trabajadores sociales gerontólogos realizan una función primordial porque poseen un conocimiento técnico-operativo sobre los sujetos y objetos de intervención, asumen el reto y la responsabilidad de sistematizar las prácticas profesionales, y contribuyen a construir el gremio profesional" (Navarro, 2016, p. 78).

las acciones desarrolladas del trabajador social requieren el apoyo del equipo interdisciplinar que entre otros aspectos debe responder a la necesidad requirente del adulto mayor, es así que para (Gil, 2012) un contexto multidisciplinario es el conjunto de disciplinas que investigan diferentes aspectos de un mismo objeto de estudio, y su interrelación es de importancia debido que permite generar conocimientos para la creación de herramientas que permitan identificar y reconocer las demandas sociales. En definitiva, los profesionales que poseen conocimientos de amplio sentido social y tienen conexión con las personas en situación de vulnerabilidad tienen como objetivo el logro de cambios significativos en el trabajo social multidisciplinario para proponer pautas y programas o proyectes basados en características que prevenir la exclusión social.

No obstante la Fundación Organización Universitaria Interamericana, menciona que “los equipos de trabajo multidisciplinario contribuyen a la creación de soluciones innovadoras, permitiendo así reconocer de forma oportuna las necesidades y problemáticas que enfrenta un grupo social" (2020, p. 8). En este sentido, la intervención de un equipo conformado por diferentes disciplinas influye a que se desarrollen propuestas innovadoras y habilidades sociales con el objeto que un comunidad, grupo o sector con problemas de exclusión participen desde una perspectiva integral en la mejora de su bienestar social.

En este sentido García (2018) establece que el trabajo multidisciplinario está formado por un grupo de profesionales de diferentes disciplinas que poseen destrezas para lograr que se cumplan los objetivos y metas comunes, además, sus labores están direccionadas a la creación de programas para satisfacer las exigencias sociales en función al bienestar de las personas que lo necesiten. Por tal motivo, el trabajo social multidisciplinario integra a personas que ejecutan actividades en diversas áreas con la finalidad que aporten conocimientos y elaboren estrategias para diseñar acciones que ayuden a intervenir de manera individual, grupal, o comunitaria en la población que afronta problemas sociales.

\section{Conclusión}

El trabajador social en el centro gerontológico Guillermina Loor de Moreno, gestiona, coordina e implementa acciones de intervención en coordinación con otros profesionales de que buscan el bienestar del adulto mayor, antes y durante el ingreso residente.

La gestión interinstitucional que realiza el Trabajo social en el centro gerontológico le permiten integrar estrategias de prevención y promoción de la salud buscando acciones integrales de manera 
paliativa, la cual sigue procesos sistemáticos de evaluación cada seis meses para identificar de manera oportuna la situación y condición del adulto mayor.

La importancia de mantener los principios sociales en las actividades de los adultos mayores, radica en el favorecimiento de la salud integral del individuo, donde el trabajador social crea programas o aplica medidas como una función protectora ante muchas enfermedades físicas y mentales, lo que contribuye a mejorar la salud y alargan el período de vida.

Este estudio tuvo limitaciones contextuales en donde la información proporcionada, al ser la institución de carácter público, se entregó de manera parcial considerando los procesos y restricciones de índole procedimental. No obstante, el apoyo que se obtuvo por otros estudios permitió abordar y culminar la investigación, a su vez permitirá que futuros investigadores tomen interés para concretar los procesos en el ámbito gerontológico de los profesionales en trabajo social.

\section{REFERENCIAS}

Cárdenas Rumazo, J., \& Cedeño Barreto, M. (2018). Intervención del trabajo social en los programas de atención al adulto mayor en los centros geriátricos en la ciudad de Portoviejo. Revista Caribeña de Ciencias Sociales. https://ng.cl/nwsft

Díaz-Tendero-Bollain, A. (2011). Estudios de Población y enfoques de Gerontología Social en México. Papeles de Población. 17(70), 49-79. https://rppoblacion.uaemex.mx/article/view/8451

Donizete Prado, S., \& Dutra Sayd, J. (2006). A gerontologia como campo do conhecimento científico: Conceito, interesses e projeto político. Ciência \& Saúde Coletiva, 11, 491-501. https://doi.org/10.1590/ S1413-81232006000200026

Filardo Llamas, C. (2011). Trabajo social para la Tercera Edad. Documentos de Trabajo Social, 49, 204-219.

Franco Narváez, V. P., \& Cárdenas-Lata, B. J. (2021). Derecho de los adultos mayores a una vida digna y la intervención de trabajo Social Comunitario.Polo del conocimiento.6(5), 18.https://polodelconocimiento. com/ojs/index.php/es/article/view/2699/html

Fundación Organización Universitaria Interamericana. (2020). Fundación Organización Universitaria Interamericana. Fundación Organización Universitaria Interamericana. https://cutt.ly/oEtkYDY

García Gutiérrez, A. Z. (2018). Vejez en Puerto Rico: Una mirada multidisciplinaria desde el Trabajo social. Voces desde el Trabajo social, 6(1), 154-173. https://doi.org/10.31919/voces.v6i1.126

Gil Rodríguez, R. (2012). Retos de la Investigación para Impulsar el Desarrollo Humano y Social. Universidad Sentimientos de la Nación A.C.

Hernández Zamora, Z. E. (2006). Cuidadores del adulto mayor residente en asilos. Index de Enfermería, $15(52-53), 40-44$.

Llarena, N., \& Hine, C. (2021). Reproductive Longevity and Aging: Geroscience Approaches to Maintain LongTerm Ovarian Fitness. The Journals of Gerontology: Series A, 76(9), 1551-156o. https://doi.org/10.1093/ gerona/glaa204

Martínez, H. D., Mitchell, M. E., \& Aguirre, C. G. (2013). Salud del Adulto Mayor - Gerontología y Geriatría. Cátedra de Medicina Preventiva y Social. https://cutt.ly/QEtlgTA

Ministerio de Inclusión Económica y Social. (2018). Centros Gerontológicos Residenciales [Gubernamental]. https://ng.cl/jvsgv

Nascimento, P. F. D. do, Sousa, I. G. D., Lopes, M. F., Marques, N. M., \& Lameira, A. P. do N. (2020). La percepción de los cuidadores familiares sobre la independencia funcional, el nivel cognitivo y el estado emocional de los ancianos. Estudos Interdisciplinares sobre o Envelhecimento, 25(1), 107-120. https://doi.org/10.22456/2316-2171.94194 
Navarro Bartels, N. (2016). Áreas de acción profesional del trabajo social gerontológico. Revista Costarricense de Trabajo social, 29, 61-74. https://revista.trabajosocial.or.cr/index.php/revista/article/view/319

Organización de Naciones Unidas. (2015, agosto 19). Los Principios de las Naciones Unidas en favor de las personas de edad. Department of Economic and Social Affairs. https://ng.cl/rzmuw

Paredes, M., \& Monteiro, L. (2019). Desde la niñez a la vejez. Teseo.

Pérez del Molino, J., \& Moya Lopez, M. (2019). Valoración Geriátrica Integral (VGI). Infogerontologia. https:// www.infogerontologia.com/vgi/index.html

Ramos-Feijóo, C., \& García, F. F. (2021). Participación en los procesos de cuidados desde el Modelo de Atención Integral y Centrada en la Persona (MAICP). Revista Prisma Social, 32, 45-68. https:// revistaprismasocial.es/article/view/4075

Sánchez Flores, F. (2019). Fundamentos epistémicos de la investigación cualitativa y cuantitativa: Consensos y disensos. Revista Digital de Investigación en Docencia Universitaria, 13(1), 102-122. https:// doi.org/10.19083/ridu.2019.644

Sanhueza Parra, M., Castro Salas, M., \& Merino Escobar, J. M. (2005). Adultos mayores funcionales: Un nuevo concepto en salud. Ciencia yenfermería, 11(2), 17-21. https://doi.org/10.4067/S0717-95532005000200004

Sepúlveda Hernández, E. (2013). Trabajo social y planificación social en el campo gerontológico, un asunto ético y político. [Congreso] III Jornadas de Trabajo Social en el Campo Gerontológico, La Plata, 30 y 31 de agosto de 2013. http://sedici.unlp.edu.ar/handle/10915/82245

Stolz, E., Hoogendijk, E. O., Mayerl, H., \& Freidl, W. (2021). Frailty Changes Predict Mortality in 4 Longitudinal Studies of Aging. The Journals of Gerontology: Series A, 76(9), 1619-1626. https://doi.org/10.1093/gerona/ glaa266

Uribe, A. F. R., Orbegozo, L. J. V., \& Linde, J. M. M. (2010). Intervención Psicológica En Adultos Mayores. Psicología desde el Caribe, 25(25), 246-258.

Varela, F. (2000). El fenómenos de la Vida. Dumont Cologne.

\section{AUTORES}

Cristhian Michael Bailón Anchundia. Egresado de la Carrera de Trabajo Social de la Facultad de Ciencias Humanísticas y Sociales de la Universidad Técnica de Manabí

Ligia Estela Loor Lino. Docente de la escuela de Trabajo Social de la facultad de Ciencias Humanísticas y Sociales de la Universidad Técnica de Manabí

\section{Conflicto de intereses}

Los autores informan que no existe conflicto de interés posible.

Financiamiento

No existió asistencia financiera de partes externas al presente artículo.

Agradecimientos 